\title{
Is the Weibel instability enhanced by the suprathermal populations or not?
}

\author{
M. Lazar, ${ }^{1, a)}$ R. Schlickeiser, ${ }^{1,2}$ and S. Poedts ${ }^{3}$ \\ ${ }^{1}$ Department of Research-Plasmas with Complex Interactions, Ruhr-Universität Bochum, \\ D-44780 Bochum, Germany \\ ${ }^{2}$ Institut für Theoretische Physik IV, Ruhr-Universität Bochum, D-44780 Bochum, Germany \\ ${ }^{3}$ Centre for Plasma Astrophysics, Celestijnenlaan 200B, 3001 Leuven, Belgium
}

(Received 30 March 2010; accepted 12 May 2010; published online 28 June 2010)

\begin{abstract}
The kinetic instabilities of the Weibel type are presently invoked in a large variety of astrophysical scenarios because anisotropic plasma structures are ubiquitous in space. The Weibel instability is driven by a temperature anisotropy which is commonly modeled by a bi-axis distribution function, such as a bi-Maxwellian or a generalized bi-Kappa. Previous studies have been limited to a bi-Kappa distribution and found a suppression of this instability in the presence of suprathermal tails. In the present paper it is shown that the Weibel growth rate is rather more sensitive to the shape of the anisotropic distribution function. In order to illustrate the distinguishing properties of this instability a product-bi-Kappa distribution is introduced, with the advantage that this distribution function enables the use of different values of the spectral index in the two directions, $\kappa_{\|} \neq \kappa_{\perp}$. The growth rates and the instability threshold are derived and contrasted with those for a simple bi-Kappa and a bi-Maxwellian. Thus, while the maximum growth rates reached at the saturation are found to be higher, and the threshold is drastically reduced making the anisotropic product bi-Kappa (with small Kappas) highly susceptible to the Weibel instability. This effect could also raise questions on the temperature or the temperature anisotropy that seems to be not an exclusive source of free energy for this instability, and definition of these notions for such Kappa distributions must probably be reconsidered. (C) 2010 American Institute of Physics. [doi:10.1063/1.3446827]
\end{abstract}

\section{INTRODUCTION}

The huge amount of incoming radiation and ionized particles from space suggests the widespread existence of kinetic anisotropies in cosmic plasmas. Moreover, space plasmas are sufficiently dilute and their collisionality is sufficiently low (see, e.g., Table 8.1 in Ref. 1) and, therefore, expected to be far from a Maxwellian equilibrium (only provided by the short-range binary collisions of plasma particles). Thus, we have direct proofs from the observations and in situ measurements that suprathermal populations are widely present at different altitudes in the solar wind plasma $^{2-5}$ and probably in the solar corona. ${ }^{6}$ These populations exhibit suprathermal high energy tails and are fitted quite well by the family of Kappa distribution functions, ${ }^{7}$ which are power laws in particle speed. The Kappa distribution generalizes the notion of equilibrium in collisionless plasmas deviated from a thermal (Maxwellian) equilibrium, and which contain fully developed turbulence in a quasistationary equilibrium. ${ }^{5,8,9}$ In such plasma systems, the temperature is redefined on the basis of a superadditive entropy. ${ }^{9,10}$

The so-called Kappa distributions are defined by using a spectral index $\kappa$, which determines the slope of the high energy tails in the velocity spectrum of plasma particles. In the limit of very large $\kappa \rightarrow \infty$, the Kappa functions degenerate into Maxwellians. ${ }^{11}$ The effects of these suprathermal populations and their anisotropies on the threshold conditions and the linear growth rates of kinetic instabilities have been studied by modeling plasma with diverse anisotropic distribu-

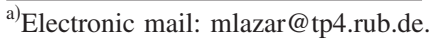

tions fully or partially populated by Kappa particles. ${ }^{12-28}$ These effects largely vary, depending on the shape of the distribution function and the nature and the frequency of the plasma mode. A selection of Kappa distributions and their limiting forms for a large $\kappa \rightarrow \infty$ is given in Ref. 11, Table I. Recent works have also introduced relativistic distributions of Kappa type for modeling the electrons and nucleons from the energetic solar events (see Ref. 29 and some references therein).

The most simple anisotropic distribution is the bi-Kappa distribution function introduced here in Eq. (1), and which describes a temperature anisotropy, $T_{\perp} \neq T_{\|}$, with the same index $\kappa_{\|}=\kappa_{\perp}=\kappa$ for both directions. This distribution has widely been used for describing kinetic instabilities in a drifting or a nondrifting plasma, e.g., the ion acoustic instability, ${ }^{12}$ the electromagnetic ion-cyclotron and firehose instabilities, ${ }^{13,14,26}$ the mirror unstable mode ${ }^{17}$ the whistler and electron cyclotron instabilities, ${ }^{15,16,19,23,25,30}$ and the nonresonant instabilities of the Weibel type. .2, $24,28^{2}$

Recently, $\mathrm{Basu}^{27}$ reviewed the stability properties of hydromagnetic waves in a plasma distributed after a productbi-Kappa function such as the one introduced here in Eq. (4). The product-bi-Kappa distribution function represents a generalization of the Kappa-Maxwellian distribution, which is a product of a one-dimensional Kappa distribution along a preferred direction in space, e.g., the guiding magnetic field, and a Maxwellian distribution in the perpendicular plane. ${ }^{18}$ In a hybrid Kappa-Maxwellian plasma, unlike a uniform Maxwellian or a uniform Kappa, the dispersion properties and the stability were found to be markedly changed. ${ }^{18,20,21}$ Notable is that because of the anisotropy of the contours in 
the velocity space, such a Kappa-Maxwellian distribution can be unstable even for equal parallel and perpendicular temperatures, or some very well-known unstable modes become stable to a temperature anisotropy in such asymmetric distributions. ${ }^{21}$

The product-bi-Kappa distribution function has not often been applied so far, but we think that this function deserves further consideration for the following reasons. (1) It decouples the dynamics of the plasma particles over the two principal directions allowing not only for distinct temperatures, $T_{\|}$and $T_{\perp}$, but for distinct spectral indices, $\kappa_{\|}$and $\kappa_{\perp}$, as well. (2) There is a possibility to regulate this coupling in accord to a guiding magnetic field or other external constraints by the interplay of $\kappa_{\|}$and $\kappa_{\perp}$. (3) Compared to a bi-Kappa, a product-bi-Kappa distribution allows for a more realistic description and (4) enlarges the number of distributions with an elaborated dispersion approach. (5) One more important feature (recently revealed for a similar KappaMaxwellian plasma $)^{21}$ is that such a product-bi-Kappa model permits further analytical progress leading to tractable expressions for the dielectric tensor elements enabling, for example, the investigation of oblique modes in a magnetized plasma.

Electromagnetic instabilities of the Weibel type ${ }^{31,32}$ are driven by an arbitrary deviation of the particle velocity distribution from its equilibrium, whether it is a bulk (relative) motion of streaming particles ${ }^{32}$ or a temperature anisotropy. ${ }^{31}$ Kinetic anisotropies are ubiquitous in space plasmas (extending from heating flows and temperature anisotropies to particle jets and shock waves in outflows, or interpenetrating plasma shells in interplanetary wind) and, presently, Weibel instabilities are invoked in a large variety of astrophysical scenarios. These instabilities create magnetic field such as the cosmological seeds necessary for the dynamo mechanism, ${ }^{33,34}$ or a quasistationary magnetic field boost for the synchrotron emissions in astrophysical sources. ${ }^{35}$ Moreover, the magnetic field fluctuations observed in interplanetary space are also attributed to these instabilities (e.g., filamentation, whistler, mirror, and oblique firehose), ${ }^{36,37}$ which compete with other constraints of plasma particles (adiabatic expansion and Coulomb collisions) and maintain a relatively small temperature anisotropy in the solar wind. ${ }^{37-41}$

Here we discuss the Weibel instability driven by a temperature anisotropy of plasma particles. Recent studies of this instability have been limited to a bi-Kappa distribution and found that the instability is suppressed in the presence of the Kappa tails. ${ }^{22,24}$ For the same arguments formulated above, we investigate here the product-bi-Kappa distribution as a favorable alternative that seems to add an excess of free energy in the velocity space and to enhance the instability. In the present paper we proceed to a comparative analysis of the effects of these two distributions, the bi-Kappa and the product bi-Kappa, on the Weibel instability. The plasma is assumed to be collisionless and spatially homogeneous.

\section{THE KAPPA DISTRIBUTIONS}

In order to describe the initially unperturbed plasma system we first introduce the bi-Kappa distribution function (a)

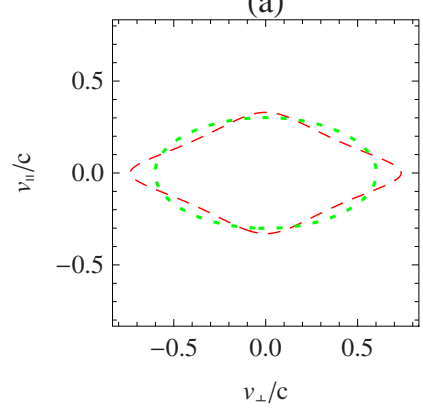

(c)

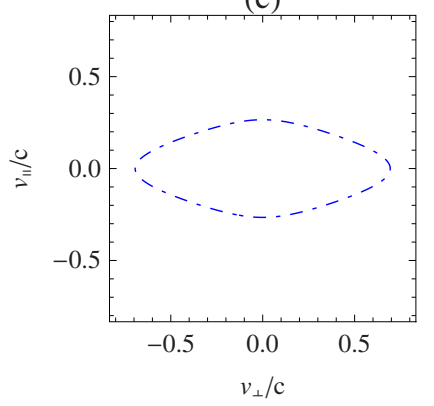

(b)

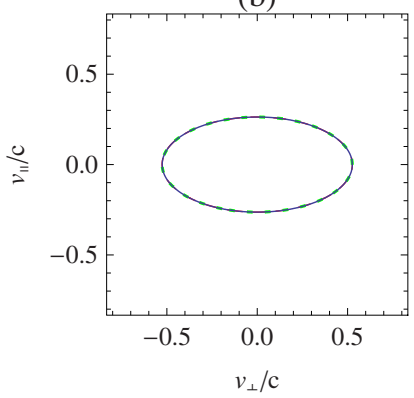

(d)

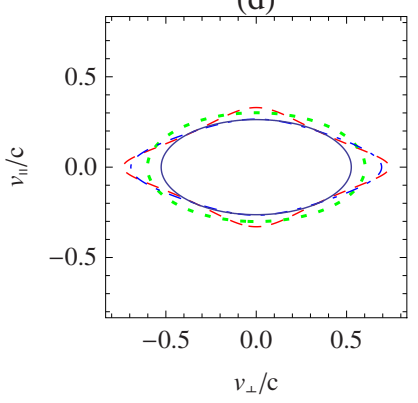

FIG. 1. (Color online) Contours of the distribution functions (1) and (4) are plotted with dotted and dashed lines, respectively, in panel (a) for $\kappa_{\|}=\kappa_{\perp}=\kappa=3$ and $v_{T_{\perp}} / c=2 v_{T_{\|}} / c=0.2$, and in (b) for a large $\kappa \rightarrow \infty$ leading to an exact fit of these two contours with that for a bi-Maxwellian (solid line). A Kappa-Maxwellian distribution function (4) with $\kappa_{\|}=3$ and $\kappa_{\perp} \rightarrow \infty$ (dotted-dashed line) is plotted in (c), and all these distributions are compared in panel (d). Remark the high energy tails for small values of $\kappa$ and the prominent anisotropy of the distribution (4).

$$
\begin{aligned}
F_{1}\left(v_{\|}, v_{\perp}\right)= & \frac{1}{\pi^{3 / 2} \theta_{\perp}^{2} \theta_{\|}} \frac{\Gamma[\kappa+1]}{\kappa^{3 / 2} \Gamma[\kappa-1 / 2]} \\
& \times\left(1+\frac{v_{\|}^{2}}{\kappa \theta_{\|}^{2}}+\frac{v_{\perp}^{2}}{\kappa \theta_{\perp}^{2}}\right)^{-\kappa-1}
\end{aligned}
$$

using polar coordinates $\left(v_{x}, v_{y}, v_{z}\right)=\left(v_{\perp} \cos \phi, v_{\perp} \sin \phi, v_{\|}\right)$ in the particle velocity space. This distribution is normalized to unity, $\int d^{3} v F_{1}=1$, and makes reference to the equivalent thermal velocities $\theta_{\|, \perp}$, which relate to the effective temperatures of the plasma particles,

$$
\begin{aligned}
& T_{\|} \equiv \frac{m v_{T_{\|}}^{2}}{2 k_{B}}=\frac{m}{k_{B}} \int d \mathbf{v} v_{\|}^{2} F_{1}\left(v_{\|}, v_{\perp}\right)=\frac{m}{2 k_{B}} \frac{2 \kappa}{2 \kappa-3} \theta_{\|}^{2}, \\
& T_{\perp} \equiv \frac{m v_{T_{\perp}}^{2}}{2 k_{B}}=\frac{m}{2 k_{B}} \int d \mathbf{v} v_{\perp}^{2} F_{1}\left(v_{\|}, v_{\perp}\right)=\frac{m}{2 k_{B}} \frac{2 \kappa}{2 \kappa-3} \theta_{\perp}^{2},
\end{aligned}
$$

for a spectral index $\kappa>3 / 2$.

The second distribution function examined here is a product bi-Kappa,

$$
\begin{aligned}
F_{2}\left(v_{\|}, v_{\perp}\right)= & \frac{1}{\pi^{3 / 2} \theta_{\perp}^{2} \theta_{\|}} \frac{\Gamma\left[\kappa_{\|}+1\right]}{\kappa_{\|}^{1 / 2} \Gamma\left[\kappa_{\|}+1 / 2\right]} \\
& \times\left(1+\frac{v_{\|}^{2}}{\kappa_{\|} \theta_{\|}^{2}}\right)^{-\kappa_{\|}-1}\left(1+\frac{v_{\perp}^{2}}{\kappa_{\perp} \theta_{\perp}^{2}}\right)^{-\kappa_{\perp}-1},
\end{aligned}
$$

as was defined by Summers and Thorne. ${ }^{11}$ This function is 

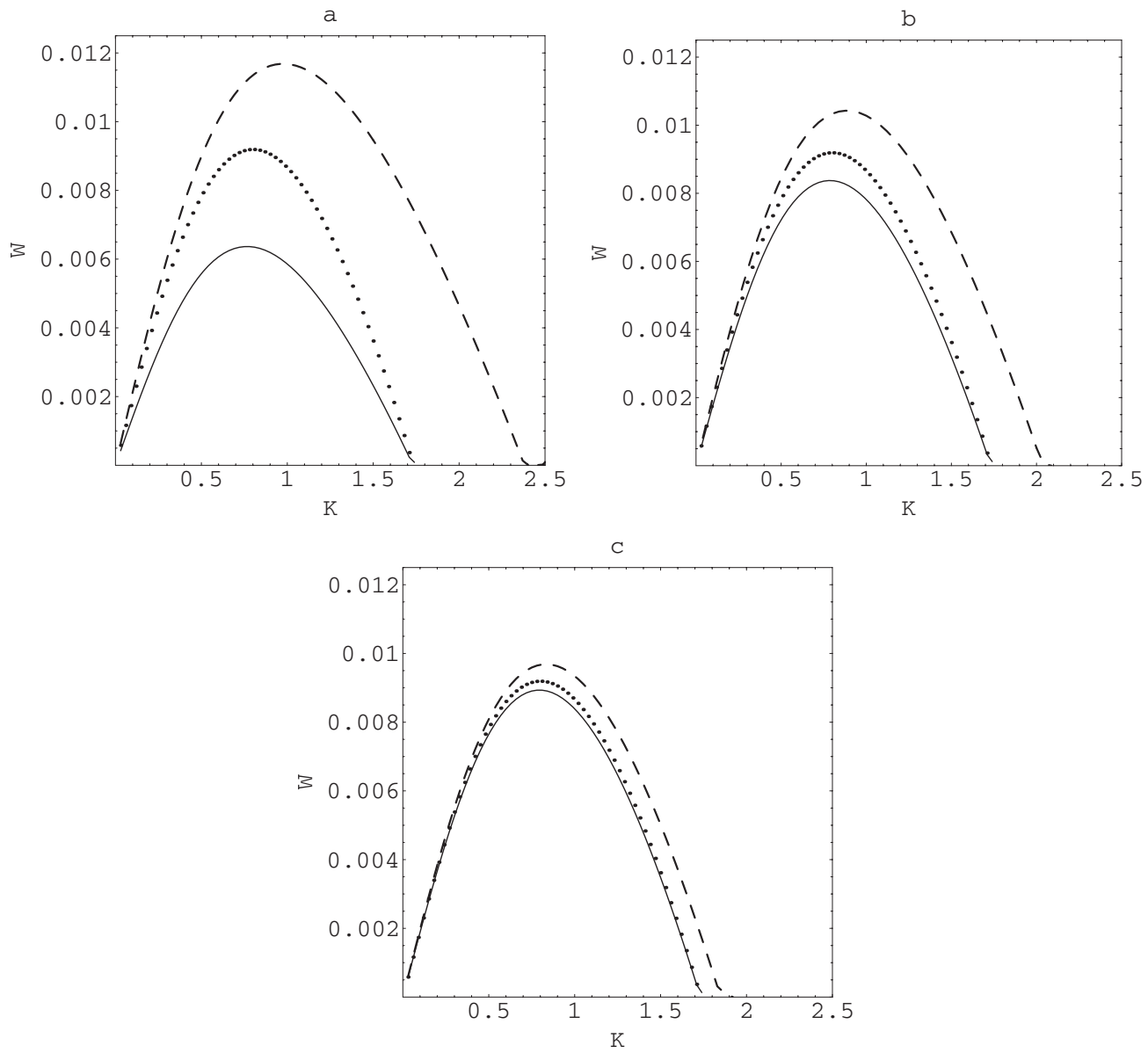

FIG. 2. The Weibel growth rates solutions of Eq. (8) (solid lines) and Eq. (11) (dashed lines) for an anisotropic Kappa distributed plasma with $v_{T_{\perp}} / v_{T_{\|}}=2$, $v_{T_{\|}}=0.02 c$, and for three values of $\kappa_{\|}=\kappa=2$ [in (a)], $\kappa=4$ [in (b)], and $\kappa=10$ [in (c)]. For a bi-Maxwellian plasma $(\kappa \rightarrow \infty)$ the growth rates are plotted with dotted lines. The coordinates are scaled as $\mathrm{W}=\omega_{i} / \omega_{p}$ and $\mathrm{K}=k c / \omega_{p}$.

also normalized to unity $\int d^{3} v F_{2}=1$ and the equivalent thermal velocities $\theta_{\|, \perp}$ are given by

$$
\begin{aligned}
& T_{\|} \equiv \frac{m v_{T_{\|}}^{2}}{2 k_{B}}=\frac{m}{k_{B}} \int d \mathbf{v} v_{\|}^{2} F_{1}\left(v_{\|}, v_{\perp}\right)=\frac{m}{2 k_{B}} \frac{2 \kappa_{\|}}{2 \kappa_{\|}-1} \theta_{\|}^{2}, \\
& T_{\perp} \equiv \frac{m v_{T_{\perp}}^{2}}{2 k_{B}}=\frac{m}{2 k_{B}} \int d \mathbf{v} v_{\perp}^{2} F_{1}\left(v_{\|}, v_{\perp}\right)=\frac{m}{2 k_{B}} \frac{\kappa_{\perp}}{\kappa_{\perp}-1} \theta_{\perp}^{2},
\end{aligned}
$$

for different spectral indices $\kappa_{\perp}>1$ and $\kappa_{\|}>1 / 2$, respectively.

Contour plots of the distribution functions (1) and (4) are displayed in Fig. 1. At low values of $\kappa$, there are significant differences between these two distributions and a prominent asymmetry of the product-bi-Kappa distribution, which indicate a surplus of temperature anisotropy, e.g., in panels (a) and (d). However, both these distribution functions approach the same bi-Maxwellian in the limit of a very large spectral index, e.g., in panel (b).

\section{THE WEIBEL INSTABILITY}

Assuming an excess of perpendicular temperature $\left(T_{\perp}>T_{\|}\right)$, the Weibel instability develops along the parallel direction (subscript "\|l") with a wave number $k=k_{\|}$, aperi- odic, $\operatorname{Re}(\omega) \equiv \omega_{r}=0$, and with a growth rate $\operatorname{Im}(\omega) \equiv \omega_{i}>0$. By using standard techniques based on the linearized Vlasov-Maxwell equations, it is easy to find the dispersion relation for the electromagnetic modes propagating along the parallel direction $^{22,24}$

$$
\frac{\omega^{2}-k^{2} c^{2}}{\omega_{p}^{2}}-1+\pi k \int_{-\infty}^{\infty} \frac{d v_{\|}}{\omega-k v_{\|}} \int_{0}^{\infty} d v_{\perp} v_{\perp}^{3} \frac{\partial F}{\partial v_{\|}}=0,
$$

where $\omega_{p}=\left(4 \pi n_{e} e^{2} / m_{e}\right)^{1 / 2}$ is the plasma frequency.

First we insert the bi-Kappa distribution function (1) in Eq. (7) and find a dispersion relation ${ }^{22,24}$

$$
\begin{aligned}
0 & =\frac{\omega^{2}-k^{2} c^{2}}{\omega_{p}^{2}}-1+\frac{\theta_{\perp}^{2}}{\theta_{\|}^{2}}\left[1+\frac{\omega}{k \theta_{\|}} Z_{\kappa}\left(\frac{\omega}{k \theta_{\|}}\right)\right] \\
& =\frac{\omega^{2}-k^{2} c^{2}}{\omega_{p}^{2}}-1+\frac{T_{\perp}}{T_{\|}}\left[1+\frac{\omega}{k \theta_{\|}} Z_{\kappa}\left(\frac{\omega}{k \theta_{\|}}\right)\right],
\end{aligned}
$$

in terms of the modified plasma dispersion function

$$
\begin{aligned}
Z_{\kappa}(f)= & \frac{1}{\pi^{1 / 2} \kappa^{1 / 2}} \frac{\Gamma[\kappa]}{\Gamma[\kappa-1 / 2]} \\
& \times \int_{-\infty}^{+\infty} d x \frac{\left(1+x^{2} / \kappa\right)^{-\kappa}}{x-f}, \quad \Im(f)>0 .
\end{aligned}
$$

This dispersion relation admits purely growing solutions of 


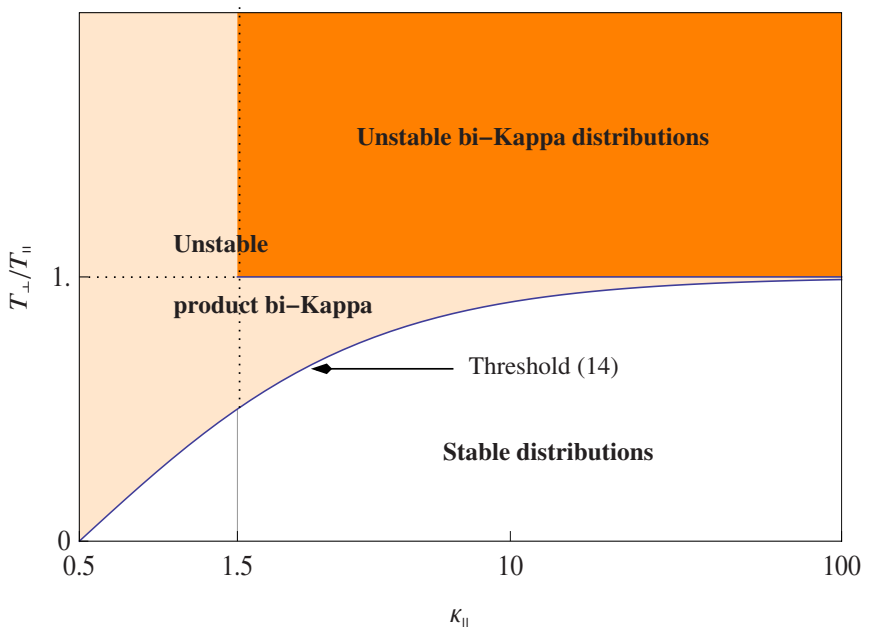

FIG. 3. (Color online) Contrast of the instability thresholds: the bi-Kappa distribution is unstable in the gray (orange online) region in the limits of $\kappa_{\|}>1.5$ and $T_{\perp} / T_{\|}>1$, and the product-bi-Kappa distribution is unstable in the light-gray (light-orange online) region limited by $\kappa_{\|}>0.5$ and condition (14) for the temperature anisotropy, and including the more restrained gray region.

the Weibel type for wave numbers smaller than a cutoff value

$$
k_{c 1}=\frac{\omega_{p}}{c}\left(\frac{\theta_{\perp}^{2}}{\theta_{\|}^{2}}-1\right)^{1 / 2}=\frac{\omega_{p}}{c}\left(\frac{T_{\perp}}{T_{\|}}-1\right)^{1 / 2} .
$$

The exact numerical values of the growth rates are displayed in Fig. 2 with solid lines for small values of $\kappa$, and with dotted lines for a very large $\kappa \rightarrow \infty$ (bi-Maxwellian plasma). In this case the cutoff wave number does not depend on the spectral index $\kappa>3 / 2$ and this is confirmed in Fig. 2 where $k_{c 1} c / \omega_{p}=\left(T_{\perp} / T_{\|}-1\right)^{1 / 2} \simeq 1.73$ is the same for all three cases. For the existence of a finite $k_{c 1} \neq 0$, the instability threshold $\tau=\min \left(T_{\perp} / T_{\|}\right)$is simply found as $T_{\perp} / T_{\|}>\tau=1$ (Fig. 3).

If we insert the second distribution function (4), the dispersion relation (7) takes a new and different form,

$$
\begin{aligned}
0 & =\frac{\omega^{2}-k^{2} c^{2}}{\omega_{p}^{2}}-1+\frac{\theta_{\perp}^{2}}{\theta_{\|}^{2}} \frac{\kappa_{\perp}\left(\kappa_{\|}+1 / 2\right)}{\kappa_{\|}\left(\kappa_{\perp}-1\right)}\left[1+\frac{\omega}{k \theta_{\|}} Z_{\kappa_{\|}}\left(\frac{\omega}{k \theta_{\|}}\right)\right] \\
& =\frac{\omega^{2}-k^{2} c^{2}}{\omega_{p}^{2}}-1+\frac{T_{\perp}}{T_{\|}} \frac{\kappa_{\|}+1 / 2}{\kappa_{\|}-1 / 2}\left[1+\frac{\omega}{k \theta_{\|}} Z_{\kappa_{\|}}\left(\frac{\omega}{k \theta_{\|}}\right)\right],
\end{aligned}
$$

where

$$
\begin{aligned}
Z_{\kappa_{\|}}(f)= & \frac{1}{\pi^{1 / 2} \kappa_{\|}^{1 / 2}} \frac{\Gamma\left[\kappa_{\|}+2\right]}{\Gamma\left[\kappa_{\|}+3 / 2\right]} \\
& \times \int_{-\infty}^{+\infty} d x \frac{\left(1+x^{2} / \kappa_{\|}\right)^{-\kappa_{\|}-2}}{x-f}, \quad \Im(f)>0 .
\end{aligned}
$$

This dispersion relation does not involve any dependence on $\kappa_{\perp}$ but only on $\kappa_{\|}$, and it can therefore be further applied to describe the instability of a Kappa-Maxwellian (onedimensional Kappa distribution in the parallel direction and a Maxwellian distribution in the perpendicular plane). Notice that both the plasma dispersion functions (9) and (12) ap- proach the standard dispersion function of Fried and Conte ${ }^{42}$ in the limit of a very large $\kappa, \kappa_{\|} \rightarrow \infty$.

The aperiodic solutions of dispersion relation (11) are displayed in Fig. 2 with dashed lines. In this case not only the growth rates increase (as was expected from Fig. 1, where the excess of anisotropy of a product-bi-Kappa distribution compared to a bi-Kappa, is evident) but the instability extends as well to larger wave numbers (smaller wavelengths) due to a non-negligible dependence on the spectral index $\kappa_{\|}$of the new cutoff wave number,

$$
k_{c 2}=\frac{\omega_{p}}{c}\left[\frac{T_{\perp}}{T_{\|}} \frac{\kappa_{\|}+1 / 2}{\kappa_{\|}-1 / 2}-1\right]^{1 / 2} .
$$

If we look, for example, at the growth rates displayed with dashed line in Fig. 2(a), and which are solutions of Eq. (11) for an index $\kappa_{\|}=\kappa=2$, and a temperature anisotropy $v_{T_{\perp}} / v_{T_{\|}}=2$ (where $v_{T_{\perp, \|}}=\sqrt{2 k_{B} T_{\perp, \|} / m}$ ), the cutoff wave number is indeed larger, $k_{c 2} c / \omega_{p}=\left[5 v_{T_{\perp}}^{2} /\left(3 v_{T_{\|}}^{2}\right)-1\right]^{1 / 2} \simeq 2.38$ $>k_{c 1} \simeq 1.73$. The cutoff wave number (13) decreases and approaches Eq. (10) only for a sufficiently large $\kappa \rightarrow \infty$.

Furthermore, for a product bi-Kappa, the anisotropy threshold $\tau$ decreases to less than unity,

$$
\frac{T_{\perp}}{T_{\|}}>\tau=\frac{\kappa_{\|}-1 / 2}{\kappa_{\|}+1 / 2}<1
$$

(Fig. 3), and toward the limit of $\kappa_{\|} \rightarrow 1 / 2$, the threshold vanishes, $\tau \sim 0$, and the instability can grow freely. If $\kappa_{\|}=2$ the instability will develop for $T_{\perp} / T_{\|}>\tau=3 / 5$, and then the instability threshold increases asymptotically to unity, $\tau \rightarrow 1$, as the spectral index becomes very large $\kappa_{\|} \rightarrow \infty$. In this brief communication we limit discussion to the example described above because it is representative for any further comparative characterization with different indices $\kappa$, temperatures, and anisotropies (in the limit of nonrelativistic values), where these Kappa distributions have shown the same effects on the Weibel instability.

\section{CONCLUSIONS}

To conclude, in this paper we revised the effects of Kappa anisotropic distributed plasmas on the Weibel instability. Previous models were limited to a bi-Kappa distribution and have been extended by introducing a more general product-bi-Kappa distribution. In this new distribution, the dynamics of plasma particles over the two principal directions are decoupled and characterized by two distinct temperatures and two distinct spectral indices, $\kappa_{\perp} \neq \kappa_{\|}$. The growth rates and the instability threshold have been found to be sensitive to the shape of the anisotropic distribution. While for a bi-Kappa distribution the Weibel instability is suppressed, for a product bi-Kappa the growth rates are enhanced and the instability threshold is significantly lowered by comparison to a Maxwellian.

The enhancing effect cannot be attributed to a resonant interaction with the energetic particles from Kappa tails because this instability is nonresonant, but it is fully supported by an excess of anisotropy and free energy of the productbi-Kappa distribution in the velocity space. In this sense, the contour plots from Fig. 1 are very suggestive: If we compare 
contours in panels (a) and (b), the bi-Kappa is less asymmetric than the bi-Maxwellian leading to lower growth rates of the Weibel instability, while the product bi-Kappa is more asymmetric than the bi-Maxwellian and enhances the instability.

The temperature anisotropy threshold for the Weibel instability can be drastically reduced in anisotropic plasmas with product-bi-Kappa distribution functions that suggest two possible explanations: either the fundamental notions, such as the temperature or the temperature anisotropy must be redefined for these Kappa distributions, or the temperature anisotropy is not an exclusive source of free energy for this instability.

These results can easily be extended to high beta plasmas ( $\beta$ is the thermal energy/magnetic energy) widely present in space and where the ambient magnetic field has only a minor influence without changing the essential features of the instability, but sustaining such asymmetric distributions with $\kappa_{\perp}>\kappa_{\|}$due to some equilibration and isotropization in the perpendicular plane, and a preferential motion and acceleration along the magnetic field.

\section{ACKNOWLEDGMENTS}

This work was supported in part by the Research Department-Plasmas with Complex Interactions, RuhrUniversität Bochum, the Katholieke Universiteit Leuven through the fellowship F/07/061, and by the Deutsche Forschungsgemeinschaft (DFG) under Grant No. Schl 201/ 21-1.

${ }^{1}$ R. Schlickeiser, Cosmic Ray Astrophysics (Springer, Heidelberg, 2002).

${ }^{2}$ W. C. Feldman, J. R. Asbridge, S. J. Bame, M. D. Montgomery, and S. P. Gary, J. Geophys. Res. 80, 4181, doi:10.1029/JA080i031p04181 (1975).

${ }^{3}$ W. G. Pilipp, H. Miggenrieder, M. D. Montgomery, K. H. Muhlhauser, H. Rosenbauer, and R. Schwenn, J. Geophys. Res. 92, 1075, doi:10.1029/ JA092iA02p01075 (1987).

${ }^{4}$ M. Maksimovic, V. Pierrard, and P. Riley, Geophys. Res. Lett. 24, 1151, doi:10.1029/97GL00992 (1997).

${ }^{5}$ L. A. Fisk and G. Gloeckler, Astrophys. J. 640, L79 (2006).

${ }^{6}$ V. Pierrard, M. Maksimovic, and J. F. Lemaire, J. Geophys. Res. 104, 17021, doi:10.1029/1999JA900169 (1999).

${ }^{7}$ V. M. Vasyliunas, J. Geophys. Res. 73, 2839, doi:10.1029/ JA073i009p02839 (1968).

${ }^{8}$ M. P. Leubner, Astrophys. Space Sci. 282, 573 (2002).

${ }^{9}$ R. A. Treumann and C. H. Jaroschek, Phys. Rev. Lett. 100, 155005 (2008).
${ }^{10}$ C. Tsallis, Physica A 221, 277 (1995).

${ }^{11}$ D. Summers and R. M. Thorne, Phys. Fluids B 3, 1835 (1991).

${ }^{12}$ D. Summers and R. M. Thorne, J. Geophys. Res. 97, 16827, doi:10.1029/ 92JA01664 (1992).

${ }^{13}$ S. Xue, R. M. Thorne, and D. Summers, J. Geophys. Res. 98, 17475, doi:10.1029/93JA00790 (1993); 101, 15457, doi:10.1029/96JA01088 (1996); 101, 15467, doi:10.1029/96JA01087 (1996).

${ }^{14}$ F. Xiao, Q. H. Zhou, H. Y. Ye, H. N. Zheng, and S. Wang, J. Geophys. Res. 112, A07219, doi:10.1029/2006JA012050 (2007).

${ }^{15}$ R. L. Mace, J. Geophys. Res. 103, 14643, doi:10.1029/98JA00616 (1998).

${ }^{16}$ F. Xiao, R. M. Thorne, and D. Summers, Phys. Plasmas 5, 2489 (1998).

${ }^{17}$ M. P. Leubner and N. Schupfer, J. Geophys. Res. 105, 27387, doi:10.1029/1999JA000447 (2000).

${ }^{18}$ M. A. Hellberg and R. L. Mace, Phys. Plasmas 9, 1495 (2002).

${ }^{19}$ A. K. Tripathi and K. D. Misra, Earth, Moon, Planets 88, 131 (2000).

${ }^{20}$ R. L. Mace and M. A. Hellberg, Phys. Plasmas 10, 21 (2003).

${ }^{21}$ T. Cattaert, M. A. Hellberg, and R. L. Mace, Phys. Plasmas 14, 082111 (2007).

${ }^{22}$ S. Zaheer and G. Murtaza, Phys. Plasmas 14, 022108 (2007).

${ }^{23}$ A. K. Tripathi and R. P. Singhal, Planet. Space Sci. 56, 310 (2008).

${ }^{24}$ M. Lazar, R. Schlickeiser, and P. K. Shukla, Phys. Plasmas 15, 042103 (2008).

${ }^{25}$ M. Lazar, R. Schlickeiser, S. Poedts, and R. Tautz, Mon. Not. R. Astron. Soc. 390, 168 (2008).

${ }^{26}$ M. Lazar and S. Poedts, Astron. Astrophys. 494, 311 (2009).

${ }^{27}$ B. Basu, Phys. Plasmas 16, 052106 (2009).

${ }^{28}$ M. Lazar, R. Tautz, R. Schlickeiser, and S. Poedts, Mon. Not. R. Astron. Soc. 401, 362 (2010).

${ }^{29}$ F. Xiao, C. Shen, Y. Wang, H. Zheng, and S. Wang, J. Geophys. Res. 113, A05203, doi:10.1029/2007JA012903 (2008).

${ }^{30}$ F. Xiao, Q. H. Zhou, H. N. Zheng, and S. Wang, J. Geophys. Res. 111, A08208, doi:10.1029/2006JA011612 (2006).

${ }^{31}$ E. S. Weibel, Phys. Rev. Lett. 2, 83 (1959).

${ }^{32}$ B. D. Fried, Phys. Fluids 2, 337 (1959).

${ }^{33}$ R. Schlickeiser and P. K. Shukla, Astrophys. J. 599, L57 (2003).

${ }^{34}$ M. Lazar, R. Schlickeiser, R. Wielebinski, and S. Poedts, Astrophys. J. 693, 1133 (2009).

${ }^{35}$ M. Medvedev and A. Loeb, Astrophys. J. 526, 697 (1999).

${ }^{36}$ A. Stockem, I. Lerche, and R. Schlickeiser, Astrophys. J. 651, 584 (2006).

${ }^{37}$ S. D. Bale, J. C. Kasper, G. G. Howes, E. Quataert, C. Salem, and D. Sundkvist, Phys. Rev. Lett. 103, 211101 (2009).

${ }^{38}$ J. C. Kasper, A. J. Lazarus, and S. P. Gary, Geophys. Res. Lett. 29, 1839, doi:10.1029/2002GL015128 (2002).

${ }^{39}$ P. Hellinger, P. Travnicek, J. C. Kasper, and A. J. Lazarus, Geophys. Res. Lett. 33, L09101, doi:10.1029/2006GL025925 (2006).

${ }^{40}$ S. Štverák, P. Travnicek, M. Maksimovic, E. Marsch, A. N. Fazakerley, and E. E. Scime, J. Geophys. Res. 113, A03103, doi:10.1029/ 2007JA012733 (2008).

${ }^{41}$ M. Lazar, S. Poedts, and R. Schlickeiser, in Proceedings of the 12th International Solar Wind Conference, 2009, edited by M. Maksimovic, K. Issautier, N. Meyer-Vernet, M. Moncuquet, and F. Pantellini (American Institute of Physics, New York, 2010), Vol. 1216, p. 280.

${ }^{42}$ B. D. Fried and S. D. Conte, The Plasma Dispersion Function (Academic, New York, 1961). 